\title{
THE PROPERTIES OF THE STELLAR NUCLEI WITH THE HOST GALAXY MORPHOLOGY IN THE ACSVCS
}

\author{
Hyun-Chul Lee \\ Department of Physics and Geology, The University of Texas-Pan American, Edinburg, TX 78539, USA \\ E-mail : leeh@utpa.edu \\ (Received May 10, 2011; Revised September 6, 2011; Accepted September 23, 2011)
}

\begin{abstract}
We have revisited the ACS Virgo Cluster Survey (ACSVCS), a Hubble Space Telescope program to obtain ACS/WFC $g$ and $z$ bands imaging for a sample of 100 early-type galaxies in the Virgo Cluster. In this study, we examine 51 nucleated early-type galaxies in the ACSVCS in order to look into the relationship between the photometric and structural properties of stellar nuclei and their host galaxies. We morphologically dissect galaxies into five classes. We note that (1) the stellar nuclei of dwarf earlytype galaxies (dS0, dE, and $\mathrm{dE}, \mathrm{N})$ are generally fainter and bluer with $g>18.95$ and $(g-z)<1.40$ compared to some brighter and redder counterparts of the ellipticals (E) and lenticular galaxies (S0), (2) the $g$-band half-light radii of stellar nuclei of all dwarf early-type galaxies (dS0, dE, and dE,N) are smaller than $20 \mathrm{pc}$ and their average is about $4 \mathrm{pc}$, and $(3)$ the colors of red stellar nuclei with $(g-z)$ $>1.40$ in bright ellipticals and lenticular galaxies are bluer than their host galaxies colors. We also show that most of the unusually "red" stellar nuclei with $(g-z)>1.54$ in the ACSVCS are the central parts of bright ellipticals and lenticular galaxies. Furthermore, we present multi photometric band color - color plots that can be used to break the age-metallicity degeneracy particularly by inclusion of the thermally pulsing-asymptotic giant branch (TP-AGB) phases of stellar evolution in the stellar population models.
\end{abstract}

Key words : Galaxies: nuclei — Stars: AGB and post-AGB — Stars: evolution

\section{INTRODUCTION}

Clear understanding of the central parts of galaxies has become an important issue in galactic and extragalactic astronomy. It appears that most galaxies have either massive black holes or stellar nuclei or both at their centers (e.g., Carollo 1999; Côté et al. 2006; Seth et al. 2010). In this context, it is highly interesting to find that there are important correlations between those massive compact central objects and their host galaxies; for instance, in terms of mass fraction (e.g., Ferrarese et al. 2006b).

In the last decade, using high resolution photometric capabilities (down to a few parsec level at the Virgo cluster distance) of the Hubble Space Telescope (HST), Côté et al. (2004) surveyed the 100 early-type galaxies in the Virgo Cluster, spanning a range of $\sim 460$ in blue luminosity, with widely separated bandpasses (F475W $\sim g$ and F850LP $\sim \mathrm{z})$. This is the ACS Virgo Cluster Survey (ACSVCS). From this ACSVCS, Côté et al. (2006) and Ferrarese et al. (2006a) showed that (1) the massive early-type galaxies with $M_{B}<-20.5$ appear to have supermassive black holes in their center, (2) about $50 \sim 80 \%$ of early-type galaxies with $-20.5<$ $M_{B}<-15$ in the Virgo cluster have stellar nuclei at or near their projected photocenters, and (3) the stellar nuclei are, on average, $3.5 \mathrm{mag}$ brighter than a typical globular cluster.
In this study, we revisit the earlier studies of the ACSVCS, especially those on stellar nuclei in the centers of the relatively low mass early-type galaxies. We dissect early-type galaxies into their morphological classes and look into the relationship between the photometric and structural properties of stellar nuclei and their host galaxies.

\section{LUMINOSITIES, COLORS, AND SIZES OF STELLAR NUCLEI OF THE ACSVCS}

Table 1 lists photometric and structural data of 51 Type Ia* stellar nuclei of the ACSVCS from Côté et al. (2006) and some properties of their host galaxies from Ferrarese et al. (2006a). We have categorized the ACSVCS early-type galaxies into five morphological classes. The five morphological classes in Table 1 are ellipticals, nucleated dwarf ellipticals (dE,N), non-nucleated dwarf ellipticals (dE), dwarf lenticulars (dS0), and lenticular galaxies (S0s), respectively, and they are listed in order of increasing $B_{T}$, the integrated $B$-band magnitude of host galaxies. Among six classes in the Table 2 of Côté et al. (2004), we have combined

\footnotetext{
* Type Ia stellar nuclei show unambiguous evidence for a nucleus at or near their photocenters and are of reliable photometric and structural parameters.
} 
Table 1.

Data of type Ia stellar nuclei of early-type galaxies in the ACSVCS

\begin{tabular}{|c|c|c|c|c|c|c|c|c|}
\hline $\mathrm{VCC}$ & Type & $B_{T}$ & $g, \mathrm{Gal}$ & $(g-z)$, Gal & $g, \mathrm{Nuc}$ & $(g-z), \mathrm{Nuc}$ & $r_{h, g}, \mathrm{Nuc}$ & Other Name \\
\hline \multicolumn{9}{|c|}{ Ellipticals } \\
\hline 828 & E5 & 12.84 & 12.71 & 1.48 & 18.53 & 1.57 & 16.64 & NGC 4387 \\
\hline 1630 & E2 & 12.91 & 12.54 & 1.50 & 17.39 & 1.68 & 40.08 & NGC 4551 \\
\hline 1146 & E1 & 12.93 & 12.70 & 1.36 & 15.37 & 1.42 & 62.40 & NGC 4458 \\
\hline 1913 & E7 & 13.22 & 12.90 & 1.43 & 17.55 & 1.60 & 47.76 & NGC 4623 \\
\hline 1422 & $\mathrm{E} 1, \mathrm{~N}:$ & 13.64 & 13.60 & 1.27 & 20.22 & 1.22 & 3.04 & IC 3468 \\
\hline 1871 & E3 & 13.86 & 14.13 & 1.45 & 18.73 & 1.25 & 10.00 & IC 3653 \\
\hline 1488 & E6: & 14.76 & 14.78 & 0.88 & 23.71 & 0.72 & 2.00 & IC 3487 \\
\hline 1545 & $\mathrm{E} 4$ & 14.96 & 14.77 & 1.34 & 21.93 & 1.05 & 4.00 & IC 3509 \\
\hline 1192 & E3 & 15.04 & 14.48 & 1.52 & 19.09 & 1.19 & 9.60 & NGC 4467 \\
\hline 1627 & E0 & 15.16 & 15.11 & 1.42 & 18.83 & 1.44 & 15.76 & \\
\hline 1440 & E0 & 15.20 & 14.67 & 1.26 & 19.70 & 1.32 & 5.04 & IC 798 \\
\hline 538 & E0 & 15.40 & 15.91 & 1.16 & 21.27 & 1.13 & 2.64 & NGC 4309A \\
\hline 1199 & E2 & 15.50 & 15.74 & 1.56 & 19.75 & 1.36 & 6.00 & \\
\hline \multicolumn{9}{|l|}{$\mathrm{dE}, \mathrm{N}$} \\
\hline 1261 & $\mathrm{~d}: \mathrm{E} 5, \mathrm{~N}$ & 13.56 & 13.29 & 1.20 & 19.50 & 1.22 & 3.28 & NGC 4482 \\
\hline 1910 & $\mathrm{dE} 1, \mathrm{~N}$ & 14.17 & 14.12 & 1.41 & 19.82 & 1.19 & 3.04 & IC 809 \\
\hline 856 & $\mathrm{dE} 1, \mathrm{~N}$ & 14.25 & 14.25 & 1.22 & 18.97 & 1.12 & 13.04 & IC 3328 \\
\hline 1355 & $\mathrm{dE} 2, \mathrm{~N}$ & 14.31 & 14.44 & 1.19 & 21.10 & 1.03 & 3.44 & IC 3442 \\
\hline 1087 & $\mathrm{dE} 3, \mathrm{~N}$ & 14.31 & 13.87 & 1.29 & 20.22 & 1.33 & 2.16 & IC 3381 \\
\hline 1861 & $\mathrm{dE} 0, \mathrm{~N}$ & 14.37 & 14.22 & 1.33 & 20.11 & 1.04 & 10.96 & IC 3652 \\
\hline 1431 & $\mathrm{dE} 0, \mathrm{~N}$ & 14.51 & 14.29 & 1.42 & 19.66 & 1.13 & 19.04 & IC 3470 \\
\hline 437 & $\mathrm{dE} 5, \mathrm{~N}$ & 14.54 & 13.82 & 1.26 & 20.00 & 1.00 & 7.12 & UGC $7399 \mathrm{~A}$ \\
\hline 2019 & $\mathrm{dE} 4, \mathrm{~N}$ & 14.55 & 14.42 & 1.19 & 20.31 & 1.12 & 2.96 & IC 3735 \\
\hline 33 & $\mathrm{~d}: \mathrm{E} 2, \mathrm{~N}:$ & 14.67 & 15.03 & 1.10 & 22.18 & 0.91 & 2.64 & IC 3032 \\
\hline 200 & $\mathrm{dE} 2, \mathrm{~N}$ & 14.69 & 14.79 & 1.21 & 22.86 & 0.92 & 4.24 & \\
\hline 1075 & $\mathrm{dE} 4, \mathrm{~N}$ & 15.08 & 14.96 & 1.20 & 21.07 & 0.96 & 3.20 & IC 3383 \\
\hline 230 & $\mathrm{dE} 4:, \mathrm{N}:$ & 15.20 & 15.45 & 1.17 & 20.31 & 1.09 & 3.04 & IC 3101 \\
\hline 2050 & dE5:,N & 15.20 & 15.12 & 1.16 & 22.38 & 0.97 & 5.84 & IC 3779 \\
\hline 1828 & $\mathrm{dE} 2, \mathrm{~N}$ & 15.33 & 15.09 & 1.25 & 21.50 & 1.00 & 4.80 & IC 3635 \\
\hline 1407 & $\mathrm{dE} 2, \mathrm{~N}$ & 15.49 & 15.03 & 1.23 & 20.39 & 0.98 & 11.60 & IC 3461 \\
\hline 1886 & $\mathrm{dE} 5, \mathrm{~N}$ & 15.49 & 15.29 & 1.00 & 22.05 & 1.01 & 2.88 & \\
\hline 1185 & $\mathrm{dE} 1, \mathrm{~N}$ & 15.68 & 15.24 & 1.28 & 20.86 & 0.95 & 4.56 & \\
\hline 1539 & $\mathrm{dE} 0, \mathrm{~N}$ & 15.68 & 15.15 & 1.21 & 20.93 & 1.11 & 18.48 & \\
\hline 1826 & $\mathrm{dE} 2, \mathrm{~N}$ & 15.70 & 15.51 & 1.16 & 20.10 & 1.19 & $(1.92)$ & IC 3633 \\
\hline 1489 & $\mathrm{dE} 5, \mathrm{~N} ?$ & 15.89 & 15.89 & 1.08 & 22.38 & 0.87 & 4.08 & IC 3490 \\
\hline 1661 & $\mathrm{dE} 0, \mathrm{~N}$ & 15.97 & 14.55 & 1.28 & 20.30 & 1.05 & 6.32 & \\
\hline \multicolumn{9}{|l|}{$\mathrm{dEs}$} \\
\hline 543 & dE5 & 14.39 & 14.12 & 1.24 & 22.56 & 1.36 & 12.56 & UGC 7436 \\
\hline 1528 & $\mathrm{~d}: \mathrm{E} 1$ & 14.51 & 14.34 & 1.29 & 22.27 & 0.96 & $(1.20)$ & IC 3501 \\
\hline 1895 & $\mathrm{~d}: \mathrm{E} 6$ & 14.91 & 14.88 & 1.11 & 23.48 & 0.88 & $(1.84)$ & UGC 7854 \\
\hline \multicolumn{9}{|l|}{ dSOs } \\
\hline 2048 & $\mathrm{~d}: \mathrm{S} 0(9)$ & 13.81 & 13.82 & 1.22 & 21.45 & 1.15 & 2.96 & IC 3773 \\
\hline 1695 & dS0: & 14.53 & 14.23 & 1.15 & 22.61 & 1.38 & $(1.76)$ & IC 3586 \\
\hline 751 & dS0 & 15.30 & 14.64 & 1.33 & 21.22 & 1.05 & 3.68 & IC 3292 \\
\hline \multicolumn{9}{|l|}{ S0s } \\
\hline 1720 & $\mathrm{~S}_{1 / 2}(4)$ & 12.29 & 11.75 & 1.44 & 18.40 & 1.57 & 6.80 & NGC 4578 \\
\hline 1619 & $\mathrm{E} 7 / \mathrm{S}_{1}(7)$ & 12.50 & 12.18 & 1.42 & 17.13 & 1.55 & 25.84 & NGC 4550 \\
\hline 1883 & $\mathrm{RSB}_{1 / 2}$ & 12.57 & 11.76 & 1.32 & 18.74 & 1.14 & $(1.92)$ & NGC 4612 \\
\hline 1242 & $\mathrm{SO}_{1}(8)$ & 12.60 & 12.19 & 1.46 & 19.84 & 1.70 & 2.80 & NGC 4474 \\
\hline 784 & $\mathrm{~S}_{1}(2)$ & 12.67 & 12.19 & 1.43 & 18.34 & 1.66 & 12.88 & NGC 4379 \\
\hline 1250 & $\mathrm{~S}_{3}(5)$ & 12.91 & 12.40 & 1.21 & 19.73 & 1.55 & 2.08 & NGC 4476 \\
\hline 1125 & $\mathrm{~S}_{1}(9)$ & 13.30 & 12.85 & 1.42 & 20.48 & 0.97 & 4.80 & NGC 4452 \\
\hline 1283 & $\mathrm{SBO}_{2}(2)$ & 13.45 & 13.02 & 1.47 & 20.65 & 1.58 & 4.24 & NGC 4479 \\
\hline 698 & $\mathrm{~S}_{1}(8)$ & 13.60 & 13.04 & 1.38 & 19.93 & 1.32 & 3.28 & NGC 4352 \\
\hline 140 & $\mathrm{SO}_{1 / 2}(4)$ & 14.30 & 14.08 & 1.22 & 22.09 & 0.91 & 2.40 & IC 3065 \\
\hline
\end{tabular}

Note.-1. Data are from Table 1 of Côté et al. (2006) and Tables 3 and 4 of Ferrarese et al. (2006a). Each category is listed in order of increasing $B_{T}$. 2. Galaxy type in the second column is from Binggeli, Sandage, \& Tammann (1985). 3. The $g$-band half-light radii of stellar nuclei in the 8 th column are in parsec and calculated with $0.1^{\prime \prime}=8$ pc. 4. Binggeli \& Cameron (1991) noted that VCC 1422 and VCC 1545 are misclassified dwarf ellipticals (see their Fig. 11 and 12). 




Fig. 1. - Stellar nuclei of Type Ia data from Côté et al. (2006) are plotted. Symbols for host galaxies are noted in the bottom right panel. Right panels additionally show the stellar nuclei of lenticular galaxies (S0).

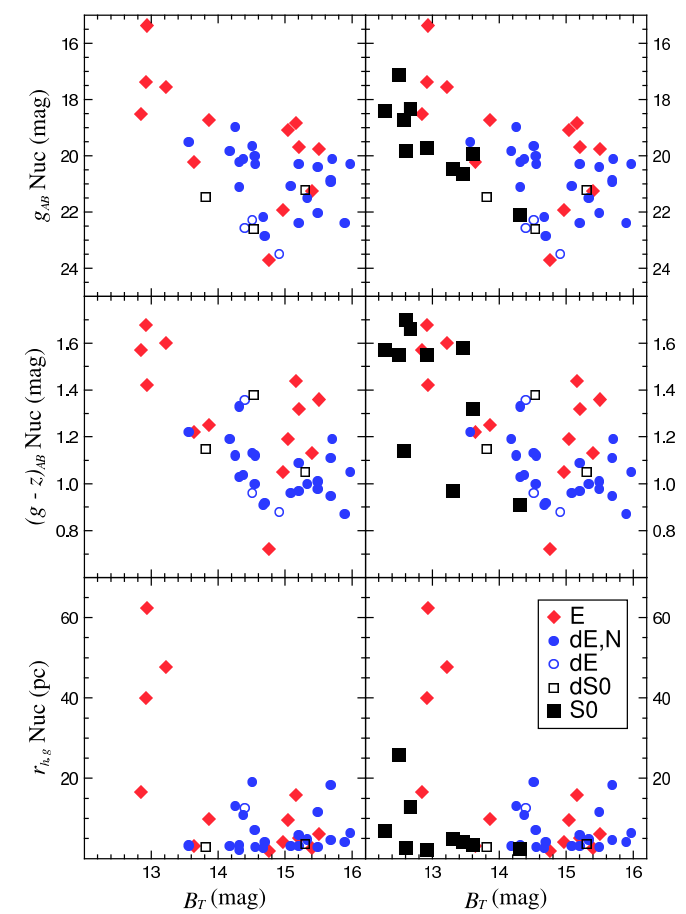

Fig. 2. - Stellar nuclei of Type Ia data from Côté et al. (2006) are plotted against the host galaxy $B$-band magnitude. Right panels additionally show the stellar nuclei of lenticular galaxies (S0). Symbols are same as in Figure 1.

$\mathrm{E} / \mathrm{S}^{\dagger}$ and $\mathrm{S} 0$ types and assigned them as $\mathrm{S} 0$ in this study.

\footnotetext{
${ }^{\dagger}$ Among 7 E/S0, only VCC 1619 is of type Ia stellar nuclei.
}

Galaxy type in the second column of Table 1 is from Binggeli, Sandage, \& Tammann (1985) 4th columns of Table 1 are the integrated magnitudes of the host galaxies in $B$ and $g$-band, respectively and the 5 th column lists the $(g-z)_{A B} \S$ of the host galaxies. The 6th, 7th and 8th columns of Table $1\left(g_{A B},(g-z)_{A B}\right.$, and $\left.r_{h, g}\right)$ are the photometric and structural properties of the stellar nuclei from Côté et al. (2006). The $g$ band half-light radii in the 8th column are calculated by adopting $0.1^{\prime \prime}=8 \mathrm{pc}$ from Côté et al. (2006) with the common distance to the Virgo cluster being 16.5 Mpc from Tonry et al. (2001). The core ellipticals with supermassive black holes are not included in this study.

The Type Ia stellar nuclei of early-type galaxies among the ACSVCS sample from Côté et al. (2006) are plotted in Fig. 1. The five classes of the earlytype galaxies are depicted here with different symbols as noted in the bottom right panel. The right panels of Fig. 1 additionally show the stellar nuclei of the lenticular galaxies (S0). In the top panels, the $(g-z)$ colors of stellar nuclei of early-type galaxies in the ACSVCS are compared with their $g$-band magnitudes and in the bottom panels the $g$-band half-light radii of the stellar nuclei are contrasted with their $g$-band magnitudes.

It is seen in the top panels of Fig. 1 that the stellar nuclei of dwarf ellipticals (dS0, dE, and dE,N) are generally fainter and bluer with $g>18.95$ and $(g-z)<$ 1.40 as compared to some brighter and redder counterparts of the ellipticals (E) and lenticular galaxies (S0). It is further noted from the top right panel of Figure 1 that six of the stellar nuclei of lenticular galaxies and three of that of ellipticals, respectively, are rather of unusual red colors with $(g-z)>1.54$. The bottom panels of Fig. 1 show that the $g$-band half-light radii of stellar nuclei of all dwarf ellipticals (dS0, dE, and $\mathrm{dE}, \mathrm{N}$ ) are smaller than $20 \mathrm{pc}$ and their average is about $4 \mathrm{pc}$.

Fig. 2 contrasts the $B_{T}$, the integrated $B$-band magnitude of host galaxies, with their stellar nuclei's $g$ band magnitues (top panels), $(g-z)$ colors (middle panels), and $g$-band half-light radii (bottom panels). Similar to Fig. 1, the right panels additionally show the stellar nuclei of lenticular galaxies (S0). It becomes clear from the right panels of Fig. 2 that the S0s are mostly brighter entities with $B_{T} \leq 13.6$ (except VCC 140 with $B_{T}=14.3$ ). There appears to be no close correlation between host galaxy luminosity and their stellar nuclei's photometric and structural parameters as suggested by Côté et al. (2006).

The colors of stellar nuclei are compared with those of their host galaxies in Fig. 3. It is seen that the colors of red stellar nuclei with $(g-z)>1.40$ in bright ellipticals and lenticular galaxies with $B_{T}<13.6$ are bluer than their host galaxies colors. On the contrary,

\footnotetext{
$¥$ According to Binggeli \& Cameron (1991), two relatively faint elliptical galaxies, VCC 1422 and VCC 1545, are misclassified dwarf ellipticals (see their Figures 11 and 12).

$\S$ The photometry is in AB system.
} 


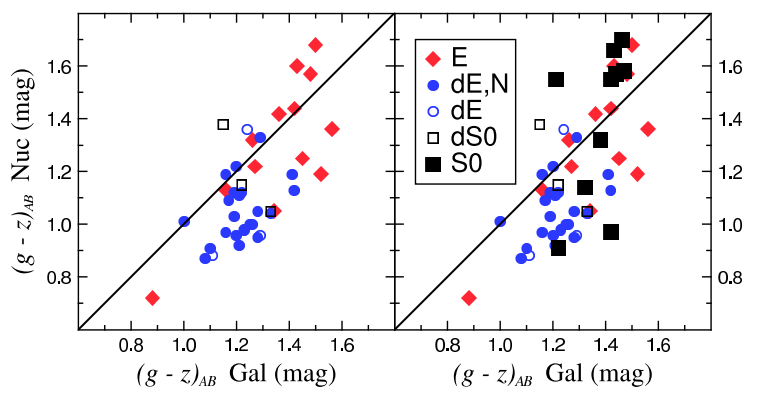

Fig. 3.- The colors of stellar nuclei are compared with that of their host galaxies. Right panels additionally show the stellar nuclei of lenticular galaxies (S0). Symbols are same as in Fig. 1.

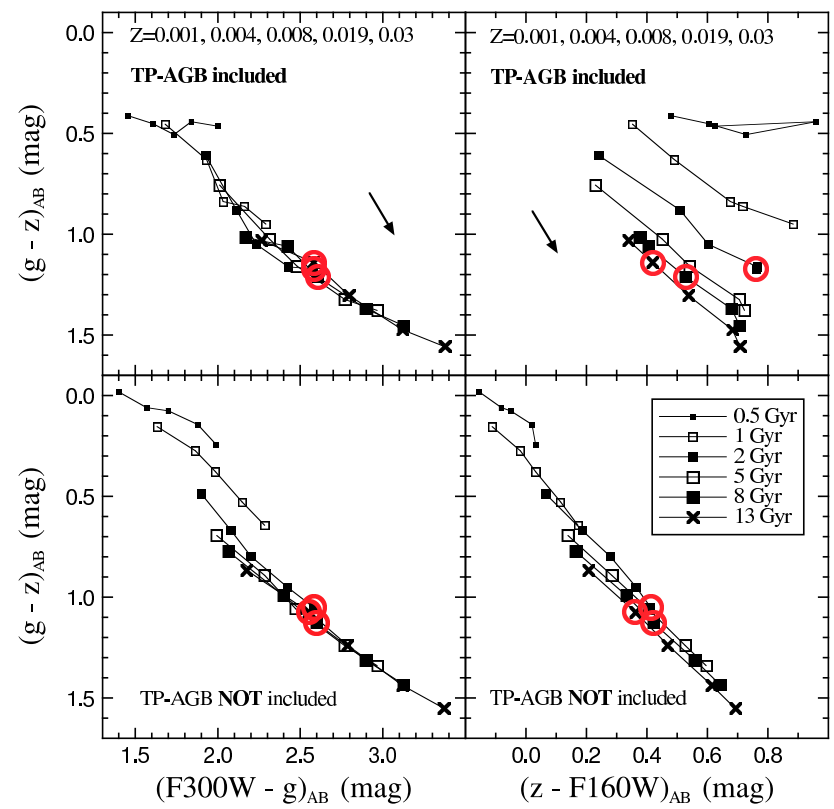

Fig. 4. - Theoretical color - color plots are shown. Top panels present models with TP-AGB stars, while bottom panels show that without TP-AGB stars (see text). Three circles in each panel demonstrate the age-metallicity degeneracy. The arrows in the top panels indicate $A_{V}=0.3 \mathrm{mag}$. At six given ages, five metallicities are connected by lines.

the colors of comparably bluer stellar nuclei with $(g-z)$ $<1.40$ in faint early-type galaxies are mostly redder than those of their host galaxies.

\section{MULTI-BAND COLOR - COLOR PLOTS}

Once we have the optical photometry ( $g$ - and $z$ band) of stellar nuclei of early-type galaxies in hand from the ACSVCS, supplementing UV (e.g., WFPC2/ F300W) and IR (e.g., NICMOS/F160W) photometry will be very useful in order to understand their star formation and chemical enrichment history. Fig. 4 shows theoretical color - color plots for the case when we have multi-band photometry. Here we have calculated the integrated simple stellar population (SSP) photometric properties by employing the latest Padova stellar models (Marigo et al. 2008) using the evolutionary population synthesis code that has been developed to study the stellar populations of star clusters and galaxies (Lee et al. 2002, 2004, 2007, 2010). Left panels are $(F 300 W-g)$ vs. $(g-z)$ and right panels are $(z-F 160 W)$ vs. $(g-z)$. Top panels present SSP models with TP-AGB stars, while bottom panels show that the same without TP-AGB stars. The arrows in the top panels indicate $A_{V}=0.3 \mathrm{mag}$. At six given ages with $0.5,1,2,5,8$, and $13 \mathrm{Gyr}$, five metallicities with $\mathrm{Z}=0.001,0.004,0.008,0.019$, and 0.03 are connected by lines. It is interesting to see from the top panels of Figure 4 that the only plausible age-metallicity combination for the $(g-z)>1.54$ that we have noted in Figure 1 is 13 Gyr and $\mathrm{Z}=0.03$.

In Fig. 4, three points are circled in order to demonstrate the well-known age-metallicity degeneracy (which is clear in the top left panel) and its potential breaking. They are $13 \mathrm{Gyr}$ with $\mathrm{Z}=0.004,8 \mathrm{Gyr}$ with $\mathrm{Z}=0.008$, and 2 Gyr with $\mathrm{Z}=0.03$, respectively. Top right panel of Fig. 4 shows that the age-metallicity degeneracy can be broken with the supplement of nearIR photometry (e.g., F160W, equivalent of $H$-band). A very old, metal-poor stellar population (13 Gyr with $\mathrm{Z}=0.004$ ) shows the same optical color as a young, metal-rich one (2 Gyr with $\mathrm{Z}=0.03)$, but the latter has a significantly redder color in the near-IR region because of the dominantly prevalent TP-AGB stars (Lee et al. 2007, 2010). Bottom right panel of Fig. 4, however, shows that the degeneracy breaking will not be possible unless the TP-AGB stars are properly engaged in the models.

\section{CONCLUSIONS AND DISCUSSION}

In this study, by employing Binggeli et al. (1985)'s morphological classification scheme within early-type galaxies, we have revisted the properties of the stellar nuclei of the early-type galaxies among the ACSVCS. We have noted that the stellar nuclei of relatively fainter early-type galaxies with $B_{T}>13.5$ are comparably fainter with $g>18.6$, bluer with $(g-z)<$ 1.45 , and smaller with $g$-band half-light radii $<20 \mathrm{pc}$ than that of the brighter early-type galaxies. Moreover, we have shown that some of the unusually "red" stellar nuclei with $(g-z)>1.54$ in the ACSVCS are the central parts of the bright ellipticals and S0 galaxies. The structural and photometric parameters for these nuclei are admittedly very hard to measure, because they are very extended and they do not have much contrast against their host galaxies. We have also presented a useful multi-band color - color plots that can potentially break the age-metallicity degeneracy with

$\Psi_{\text {http://stev.oapd.inaf.it/cgi-bin/cmd }}$ 
the addition of near-IR photometry once the TP-AGB stars are properly employed in the models.

It is tempting to speculate that the stellar nuclei are the merger products of globular clusters (GCs) by dynamical friction (e.g., Tremaine et al. 1975; Oh \& Lin 2000; Lotz et al. 2001; Capuzzo-Dolcetta \& Miocchi 2008; Agarwal \& Milosavljević 2010). The VCC 1422, VCC 856, VCC 1087, VCC 1545, VCC 1407, and VCC 1539's stellar nuclei clearly show comparatively larger sizes than that of their GCs within them (Fig. 25, 29, 30, 39, 46, and 48 of Ferrarese et al. 2006a, respectively). The larger dwarf elliptical galaxies (e.g., VCC 1261, VCC 1910, VCC 1087, VCC 1431) are presumably of the brighter and redder stellar nuclei in the upper left panel of Fig. 1 because they are perhaps the merger products of several massive red star clusters that used to inhabit the region near the center. Maybhate et al. (2010) recently note that the number of red globular clusters associated with bulges in early-type spirals generally increases with the bulge luminosity similar to elliptical galaxies (e.g., Rhode \& Zepf 2004; Peng et al. 2005).

\section{ACKNOWLEDGMENTS}

The author is grateful to J. Blakeslee, E. Grebel, J. Kormendy, T. Lisker, and C. J. Walcher for a very useful and insightful discussion. The author also thanks to the anonymous referee for her/his thoughtful comments that improved this presentation. Support for Program number HST-GO-11083 was provided by NASA through a grant from the Space Telescope Science Institute, which is operated by the Association of Universities for Research in Astronomy, Incorporated, under NASA contract NAS5-26555.

\section{REFERENCES}

Agarwal, M., \& Milosavljević, M. 2011, Nuclear Star Clusters from Clustered Star Formation, ApJ, 729, 35

Binggeli, B., \& Cameron, L. M. 1991, Dwarf Galaxies in the Virgo Cluster. I - The Systematic Photometric Properties of Early-Type Dwarfs, A\&A, 252, 27

Binggeli, B., Sandage, A., \& Tammann, G. A. 1985, Studies of the Virgo Cluster. II - A catalog of 2096 galaxies in the Virgo Cluster area, AJ, 90, 1681

Capuzzo-Dolcetta, R., \& Miocchi, P. 2008, SelfConsistent Simulations of Nuclear Cluster Formation through Globular Cluster Orbital Decay and Merging, MNRAS, 388, L69

Carollo, C. M. 1999, The Centers of Early- to Intermediate-Type Spiral Galaxies: A Structural Analysis, ApJ, 523, 566

Côté, P., Blakeslee, J. P., Ferrarese, L., et al. 2004, The ACS Virgo Cluster Survey. I. Introduction to the Survey, ApJS, 153, 223
Côté, P., Piatek, S., Ferrarese, L., et al. 2006, The ACS Virgo Cluster Survey. VIII. The Nuclei of EarlyType Galaxies, ApJS, 165, 57

Ferrarese, L., Côté, P., Jordán, A., et al. 2006a, The ACS Virgo Cluster Survey. VI. Isophotal Analysis and the Structure of Early-Type Galaxies, ApJS, 164,334

Ferrarese, L., Côté, P., Dalla Bontà, E., et al. 2006b, A Fundamental Relation between Compact Stellar Nuclei, Supermassive Black Holes, and Their Host Galaxies, ApJ, 644, L21

Lee, H.-C., Gibson, B. K., Flynn, C., Kawata, D., \& Beasley, M. A. 2004, Is the Initial Mass Function of Low Surface Brightness Galaxies Dominated by Low-Mass Stars?, MNRAS, 353, 113

Lee, H.-C., Lee, Y.-W., \& Gibson, B. K. 2002, Horizontal-Branch Morphology and the Photometric Evolution of Old Stellar Populations, AJ, 124, 2664

Lee, H.-C., Worthey, G., \& Blakeslee, J. P. 2010, Effects of alpha-Element Enhancement and the Thermally Pulsing-Asymptotic Giant Branch on Surface Brightness Fluctuation Magnitudes and Broadband Colors, ApJ, 710, 421

Lee, H.-C., Worthey, G., Trager, S. C., \& Faber, S. M. 2007, On the Age and Metallicity Estimation of Spiral Galaxies Using Optical and Near-Infrared Photometry, ApJ, 664, 215

Lotz, J. M., Rosemary, T., Ferguson, H. C., et al. 2001, Dynamical Friction in DE Globular Cluster Systems, ApJ, 552, 572

Marigo, P., Girardi, L., Bressan, A., et al. 2008, Evolution of Symptotic Giant Branch Stars. II. Optical to Far-Infrared Isochrones with Improved TP-AGB Models, A\&A, 482, 883

Maybhate, A., Goudfrooij, P., Chandar, R., \& Puzia, T. H. 2010, The Formation of Spheroids in Earlytype Spirals: Clues from Their Globular Clusters, ApJ, 721, 893

Oh, K. S., \& Lin, D. N. C. 2000, Nucleation of Dwarf Galaxies in the Virgo Cluster, ApJ, 543, 620

Peng, E. W., Jordán, A., Côté, P., et al. 2006, The ACS Virgo Cluster Survey. IX. The Color Distributions of Globular Cluster Systems in Early-Type Galaxies, ApJ, 639, 95

Rhode, K., \& Zepf, S. E. 2004, The Globular Cluster Systems of the Early-Type Galaxies NGC 3379, NGC 4406, and NGC 4594 and Implications for Galaxy Formation, AJ, 127, 302

Seth, A. C., Cappellari, M., Neumayer, N., et al. 2010, The NGC 404 Nucleus: Star Cluster and Possible Intermediate-mass Black Hole, ApJ, 714, 713

Tonry, J. L., Dressler, A., Blakeslee, J. P., et al. 2001, The SBF Survey of Galaxy Distances. IV. SBF Magnitudes, Colors, and Distances, ApJ, 546, 681 
Tremaine, S. D., Ostriker, J. P., \& Spitzer, L., Jr. 1975,

The Formation of the Nuclei of Galaxies. I - M31,

ApJ, 196, 407 